\title{
Hypogammaglobulinemia in sub-Saharan Africa: a case report and review of the literature
}

\author{
Jennifer Hsu ${ }^{1}$, Robert Opoka ${ }^{2}$, Troy C Lund ${ }^{3}$
}

1. Mayo Medical School, Rochester, Minnesota

2. Department of Pediatrics, Makerere University, Kampala, Uganda

3. Department of Pediatrics, Division of Hematology, Oncology and BMT, University of Minnesota, Minneapolis, Minnesota

\begin{abstract}
Patients with hypogammaglobulinemia are susceptible to recurrent bacterial, viral, fungal, and parasitic infections. The most common clinical manifestation includes recurrent severe infections caused by encapsulated bacteria, in which antibody opsonization is the primary defense mechanism. To our knowledge, this is the first case report of hypogammaglobulinemia in a Ugandan child in Sub-Saharan Africa. The case emphasizes the importance of including hypogammaglobulinemia in the differential diagnosis for children presenting with a history of recurrent infections.
\end{abstract}

Aim: To raise the index of clinical suspicion of hypogammaglobulinemia in an African child and allow for prompt recognition and management of hypogammaglobulinemia.

Keywords: hypogammaglobulinemia, recurrent infections, Uganda

DOI: http://dx.doi.org/10.4314/ahs.v15i1.41

\section{Introduction}

The most common worldwide causes of immunodeficiency include HIV, malnutrition, and immunosuppressive agents. However, when HIV infection, malnutrition, and other secondary causes of hypogammaglobulinemia have been excluded, it is important to consider a primary cause of hypogammaglobulinemia. Primary humoral immunodeficiencies are characterized by recurrent, severe upper and lower respiratory tract infections due to encapsulated bacteria. Although the incidence of hereditary immunodeficiency is low when compared to acquired immunodeficiency, the prevalence of people affected by a primary immunodeficiency is as high as 1 in 500 persons $^{1}$. We report a case of a 9 year old Ugandan boy with the diagnosis of hypogammaglobulinemia.

\section{Corresponding author:}

Troy C Lund

University of Minnesota

Department of Pediatrics,

Division of Hematology, Oncology and BMT

420 Delaware St. SE, Minneapolis, Minnesota 55905

Phone: (612) 625-4185

Email: lundx072@umn.edu

\section{Case Report}

A 9-year-old African boy first presented to Mulago hospital in Kampala, Uganda at the age of 5 years with increasingly frequent and severe recurrent respiratory tract infections since infancy. The infections started with a measles-like illness at 3 months of age, which had responded to broad spectrum antibiotics. He developed bacterial meningitis at the age of two, which was successfully treated. He has had a history of recurrent severe pneumonia's approximately two per year since age 2 .

At age 4, chest radiograph and computer tomography of the chest revealed multi-segmental consolidation and collapse of the right middle lobe. He was given several courses of antibiotics with no improvement in symptoms. The recurrent episodes of pneumonia persisted. Six months later the patient underwent a right middle lobectomy for persistent pneumonia unresponsive to medical treatment. Pathology revealed localized bronchiectasis with atelectasis and negative cultures. Despite the lobectomy, the recurrent respiratory tract infections did not subside.

After the lobectomy, he required several hospitalizations, including episodes of septic arthritis of the left knee joint and of pyogenic meningitis. Repeated HIV testing on the child and parents were negative. Immunoglobulin studies were sent to the USA to aid in di- 
agnosis. Rate nelphelometry was used to measure Ig concentrations on a Beckman Coulter IMMAGE® 800 Analzyer. It revealed deficiency of immunoglobulins $\mathrm{IgG}, \mathrm{IgM}$, and $\mathrm{IgA}$ with values of $28 \mathrm{mg} / \mathrm{dL}$ (normal
$565-1765 \mathrm{mg} / \mathrm{dL}$ ) $14 \mathrm{mg} / \mathrm{dL}$ (normal $55-375 \mathrm{mg} / \mathrm{dL}$ )

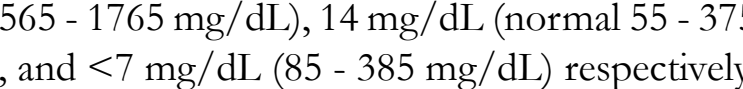

On physical exam, he was small for his age, but otherwise in fair condition. He had somewhat labored breathing and coarse crepitations, more marked on the right upon posterior chest auscultation. Heart sounds were normal with no murmurs. There was no cervical, supraclavicular, or inguinal lymphadenopathy. Abdomen showed no splenomegaly or masses. Skin was free of neurocutaneous stigmata, rashes or bruises. Neurologic exam was intact.

This child's extensive history of chronic infections, physical exam findings, imaging studies, and lab tests suggest a diagnosis of hypogammaglobulinemia. The child was given Septra for prophylaxis, since IVIG was too costly and not available. The child is alive to date.

\section{Discussion}

While normal healthy children may have up to six uppe respiratory tract infections in a given year ${ }^{2}$, infection will normally either clear relatively quickly or respond well to antibiotics. Patients with hypogammaglobulinemia are susceptible to recurrent and severe bacterial, viral, fungal, and parasitic infections. Symptoms typically begin around 6 months of age when concentrations of maternal $\mathrm{IgG}$ antibodies decrease.

Children often have associated findings of failure to thrive, recurrent fevers, and chronic diarrhea. Because of a decrease in immunoglobulins and thus decrease in antibody oposinization, patients with hypogammaglobulinemia have an increased susceptibility to encapsulated bacteria. Patients present with sepsis, septic arthritis, CNS infections, and osteomylelitis due to encapsulated organisms . Gastroenteritis infections are also commonly caused by Salmonella and Campylobacter. Patients may also present with severe hepatitis B, viral encephalitis, and bacterial meningitis ${ }^{3}$. Roughly two-thirds of patients affected with primary hypogammaglobulinemia will have lymphoid hyperplasia manifesting in the liver, spleen, tonsils, or peripheral nodes ${ }^{4}$. Because of chronic immune activation, hepatosplenomegaly may be present. Findings of hypoplasia in tissue usually rich in B lymphocytes will be present in patients with $\mathrm{X}$-linked gammaglobulinemia. Autoimmune diseases, including diabetes mellitus, rheumatoid arthritis, and inflam y bowel disease, have been observed in patients with hypogammaglobulinemia.

All forms of hypogammaglobuinemia have similar clincal presentations and are primarily distinguished from each other based on Ig levels. X-linked agammaglobulinemia (XLA) initially begins between 6 to 18 month of age and is due to a defect in Bruton's tyrosine kinase. Patients present with recurrent bacterial and respiratory tract infections. Hyper-IgM syndrome (HIGM) occurs in those with deficient $\operatorname{IgG}, \operatorname{Ig} A$, and $\operatorname{IgE}$ levels and normal or high levels of IgM. Transient hypogammaglobulinemia of infancy occurs in infants who initially have a low Ig level that eventually will increase to normal Ig levels.

Early diagnosis and treatment is essential to the quality of life and survival of the patient. Diagnosis is based on a careful history, family history, and physical exam. Laboratory tests that should be obtained if a primary humoral immunodeficiency is suspected include a complete blood count with differential, quantitative serum immunoglobulin levels ( $\operatorname{IgG}, \operatorname{IgA}$, and $\operatorname{IgM}$ ), and measurement of antibody titers to vaccines ${ }^{5}$. Treatment options include immune globulin replacement therapy which is very effective in reducing bacterial infections, though of limited availability in sub-Saharan Africa. Two forms of immune globulin therapy available include intravenous (IVIG) and subctuaneous (SCIG). The standard initial dose of IVIG is between 300 to 500 $\mathrm{mg} / \mathrm{kg}$ every three to four weeks. Dosing is determined

based on the patient's weight, trough levels of $\mathrm{IgG}$ after treatment has started, and the clinical response of the patient after treatment. Not only is an accurate diagnosis important for the patient, but it is also importan for the patient's family members who may be female carriers of the disease.

\section{Review of Literature}

A literature review shows no other reported cases of $\mathrm{X}$-linked hypogammaglobulinemia reported in Sub-Saharan Africa. This is a case of a 9-year-old boy in sub-saharan Africa, who presented with frequent and recurrent respiratory tract infections since infancy. Based on immunoglobulin level studies, it was determined that he had X-linked hypogammaglobulinemia. Limitations in making the diagnosis in sub-sarahan Africa include lack of resources available for immunoglobulin testing and potential lack of awareness of the disease. However, with this case report, we hope to make the diagnosis of X-linked hypogammaglobulinemia more known in sub-saharan Africa.

Author contribution

Each author contributed equally to this case report.

Conflict of interest

None of the authors have a conflict of interest.

\section{Source of funding}

There was no funding source for this case report.
References

Mendiratta V, Jabeen M. Infantile hemangioma: An update. Indian J Dermatol Venereol Leprol 2010;76:469-75

2. Durairaj VD. Treatment of deep orbital hemangiomas of infancy: an overview. Arch Facial Plast Surg. 2006;8:217-20.

3. Freidman, J.N., Kaiser, K.P. (2007) Essentials of ophthalmology. London: Saunders

4. Shields JA, Shields CL. Orbital cysts of childhood--classification, clinical features, and management Surv Ophthalmol. May-Jun 2004;49:281-99.

5. Cavazza S, D’Ottavio G, Pensiero S et al. Congenita dacryocystocele: diagnosis and treatment. Acta Otorhinolaryngol Ital 2008;28:298-301. 